\title{
Effect of feeding pattern on infant illness in Chinese cities
}

\author{
Li Cai $^{1}$ †, Pan Yu ${ }^{1} \neq$, Yumei Zhang ${ }^{1, *}$, Xiaoguang Yang ${ }^{2}$, Wenjun $\mathrm{Li}^{3}$ and Peiyu Wang ${ }^{1}$ \\ 'Department of Nutrition and Food Hygiene, School of Public Health, Peking University Health Science Center, Room \\ 415, 38 Xueyuan Road, Haidian District, Beijing 100191, People's Republic of China: ${ }^{2}$ National Institute for \\ Nutrition and Health, Chinese Centre for Disease Control and Prevention, Beijing, People's Republic of China: \\ ${ }^{3}$ Nestlé Nutrition Institute of China, Beijing, People's Republic of China
}

Submitted 27 March 2015: Final revision received 3 August 2015: Accepted 11 August 2015: First published online 2 September 2015

\begin{abstract}
Objective: To investigate the effect of different feeding patterns on the occurrence of diseases among infants.

Design: Data on socio-economic status, feeding patterns before 6 months (exclusive breast-feeding (EBF); mixed feeding with breast milk and formula (MBF); exclusive formula-feeding (EFF)) and illness of infants were collected via face-to-face interviews. The proportions of infants who had ever been ill or hospitalized and their potential influence factors were investigated.

Setting: Eight large cities in China.

Subjects: Infants ( $n$ 1654) aged 0-11.9 months were recruited from hospitals.

Results: For infants aged 0-2.9 months, the percentage who had been ill was $19 \cdot 2 \%, 24 \cdot 1 \%$ and $26 \cdot 3 \%$ among the EBF, MBF and EFF groups, respectively. For those aged 3-5.9 and 6-11.9 months, the corresponding percentages were $41.6 \%$, $45.6 \%$ and $51.0 \%$, and $67.0 \%, 73.4 \%$ and $67.7 \%$. Respiratory disease was the most common reported illness and cause of hospitalization. The risks of having (total) illness, diarrhoea and respiratory disease increased significantly with age, but not allergic disease. Compared with EBF, MBF and EFF infants had significantly higher risks of having illnesses except for allergic disease, and feeding patterns were not related to hospitalization. Low birth weight, middle family income and low level of mother's education also increased the risk of illness.

Conclusions: A protective effect of EBF against total illness in urban Chinese infants was found. An increasing trend with age was observed among the percentages of infants who had been ill or had diarrhoea or respiratory disease, but not allergic disease.
\end{abstract}

Keywords
Feeding patterns
Infants
Illness
China

Breast milk is the ideal source of infant nutrition mostly because of its nutritional, immunological and psychological benefits ${ }^{(1)}$. Breast milk contains thousands of components $^{(2)}$ that are necessary for infant development. In particular, it can help build the infant's immune system and prevent diseases and infections, as well as having long-term consequences for metabolism and diseases later in life ${ }^{(3)}$.

Formula milk as a product of advanced technology has been formulated to imitate breast milk, but the former does not have the same effects on babies as the latter. Different feeding patterns, such as exclusive breastfeeding (EBF), mixed feeding with breast milk and infant

$†$ Current address: Department of Maternal and Child Health, School of Public Health, Sun Yat-Sen University, Guangzhou, Guangdong, People's Republic of China.

† Co-first author. formula (MBF) and exclusive formula-feeding (EFF), have different effects on infants. Previous studies have suggested that breast-feeding, including EBF, predominant breast-feeding for at least 6 months and partial breast-feeding for up to 1 year, may reduce the risk of diarrhoea, respiratory illness and other common diseases in infants ${ }^{(4-9)}$. However, for allergic diseases, such as wheezing/asthma, eczema, allergic rhinitis and atopic dermatitis, whether breast-feeding is protective or a risk remains controversial ${ }^{(10-13)}$.

In China, previous studies have investigated the association of feeding pattern and different infant illnesses. However, most of these studies focused on clinical cases only and recruited subjects from a single hospital ${ }^{(8,14,15)}$. In the present study, the participants were selected from the general infant population aged 0-11.9 months in eight cities, regardless of whether 
they had ever had a disease or been hospitalized for treatment. We aimed to investigate the effect of different feeding patterns on the occurrence of diseases in Chinese infants.

\section{Methods}

\section{Participants}

Infants aged 0-11.9 months were included in the present study, which is part of the Maternal Infant Nutrition and Growth (MING) study. The MING study is a crosssectional study that was conducted between October 2011 and March 2012. A multiple stratified sampling design was applied. In the first stage, eight Chinese cities, namely Beijing, Shanghai, Guangzhou, Chengdu, Shenyang, Suzhou, Zhengzhou and Lanzhou, were selected via a purpose sampling method. In the second stage, two maternal and child health hospitals in each city were randomly selected. In the third stage, infants were recruited from out-patient clinics (Department of Child Care) when they returned to the hospitals for routine follow-up visits and they were not hospitalized. These infants were stratified by age group (0-2.9, 3-5.9 and 6-11.9 months) and feeding pattern (for those infants aged 6 months and below) when they came for the follow-up visit, and we recruited every fifth infant in each group. A total of 1654 infants were included, with details as follows: 671 aged 0-2.9 months (255 for EBF, 237 for MBF and 179 for EFF), 501 aged 3-5.9 months (185 for EBF, 171 for MBF and 145 for EFF) and 482 aged 6-11.9 months.

\section{Data collection}

In each selected hospital, several trained medical researchers administered structured questionnaires to obtain participant information during face-to-face interviews at the Department of Child Care. The collected information included the following: (i) general information including the infant's age, sex, ethnicity, birth weight and length, delivery mode, parents' education level and per capita family income; (ii) physical information; and (iii) dietary information. Parents' education level was assessed using a categorical scale of 'middle school or lower', 'high school', 'junior college' and 'college and above'. Per capita family income was also assessed using the categorical scale of ' $\leq 1500$ RMB', '1501-2000 RMB', '2001-3000 RMB', '3001-4000 RMB', ‘4001-6000 RMB' and '> 6000 RMB'.

Information on infant illness was determined by the following questions. Caregivers were asked, 'Has your baby ever been ill since s/he was born?' Those answered 'yes' were asked, 'The disease s/he had was (i) diarrhoea; (ii) respiratory disease (cold, bronchitis, pneumonia, etc.); (iii) allergic disease (eczema, atopic dermatitis, rhinitis, asthma, etc.); and (iv) others'. Caregivers were allowed to select as many as were applicable. Information on infant hospitalization was determined by the following questions. Caregivers were asked, 'Has your baby ever been hospitalized for treatment of disease since s/he was born?' Those answered 'yes' were asked, 'The cause for hospitalization was (i) diarrhoea; (ii) respiratory disease (cold, bronchitis, pneumonia, etc.); (iii) allergic disease (eczema, atopic dermatitis, rhinitis, asthma, etc.); and (iv) others'. Caregivers were also allowed to select all applicable cases.

\section{Definitions}

In the present study, EBF refers to an infant being fed breast milk as the only source of milk and not infant formula or animal milk, with or without water, vitamin supplements, prescribed medicines and solids.

For infants aged 6 months and below, they were considered EBF if they had been exclusively breast-fed since they were born, MBF if they had been fed with both breast milk and infant formulas since they were born, or EFF if they had been fed exclusively with infant formulas since they were born.

For infants aged 6-11.9 months, they were considered EBF if they had been exclusively breast-fed before 6 months of age, MBF if they had been fed with both breast milk and infant formulas before 6 months of age, or EFF if they had been fed exclusively with infant formulas before 6 months of age.

The infants' birth weights were classified into three categories: low birth weight $(<2.5 \mathrm{~kg})$, adequate birth weight $(2 \cdot 5-3.9 \mathrm{~kg})$ and macrosomia $(\geq 4.0 \mathrm{~kg})$.

\section{Statistical analyses}

The proportions of infants' illness and hospitalization (including total illness, diarrhoea, respiratory disease and allergic disease) were calculated by feeding pattern and age group. The differences in proportions among different feeding patterns (EBF, MBF and EFF) were determined using both the trend $\chi^{2}$ test and $\chi^{2}$ test. For the proportions of infants' illness, subgroup analyses by infants' sex, birth weight, delivery mode, their mothers' education and per capita monthly income were also performed.

Multiple logistic regression models were used to investigate the potential influence factors for infants' illness. Dependent variables included total illness, diarrhoea, respiratory disease, allergic disease and (total) hospitalization. Independent variables included in the models were infants' feeding pattern, age group, sex, birth weight, delivery mode, their mothers' education and per capita monthly income.

The statistical software package SAS version 9.2 was used for statistical analyses. Two-tailed $P<0.05$ was considered statistically significant. 
Table 1 Feeding mode and proportion of illness in infants aged 0-2.9 months from eight large cities in China, Maternal Infant Nutrition and Growth (MING) study, October 2011-March 2012

\begin{tabular}{|c|c|c|c|c|c|c|c|c|c|c|c|c|c|}
\hline & \multirow[b]{2}{*}{$n$} & \multicolumn{3}{|c|}{ Total illness (\%) } & \multicolumn{3}{|c|}{ Diarrhoea (\%) } & \multicolumn{3}{|c|}{ Respiratory disease (\%) } & \multicolumn{3}{|c|}{ Allergic disease (\%) } \\
\hline & & EBF & MBF & EFF & EBF & MBF & EFF & EBF & MBF & EFF & EBF & MBF & EFF \\
\hline Total & 671 & $19 \cdot 2$ & $24 \cdot 1$ & $26 \cdot 3$ & $5 \cdot 1$ & $4 \cdot 2$ & 4.5 & $8 \cdot 2$ & $10 \cdot 6$ & $13 \cdot 4$ & $5 \cdot 1$ & 8.9 & 9.5 \\
\hline \multicolumn{14}{|l|}{ Sex } \\
\hline Girls & 309 & $19 \cdot 7$ & $26 \cdot 8$ & 27.5 & 2.6 & 5.4 & $3 \cdot 8$ & $11 \cdot 1$ & $10 \cdot 7$ & 12.5 & $6 \cdot 0$ & 11.6 & $10 \cdot 0$ \\
\hline Boys & 359 & $19 \cdot 0$ & $22 \cdot 0$ & $25 \cdot 3$ & $7 \cdot 3$ & 3.3 & $5 \cdot 1$ & $5 \cdot 8$ & $10 \cdot 6$ & $14 \cdot 1^{*}$ & 4.4 & $6 \cdot 5$ & $9 \cdot 1$ \\
\hline \multicolumn{14}{|l|}{ Birth weight } \\
\hline Low birth weight & 17 & $25 \cdot 0$ & $25 \cdot 0$ & $66 \cdot 7$ & 0.0 & 0.0 & 0.0 & 0.0 & 0.0 & $55 \cdot 6^{\star}, \dagger$ & 0.0 & $25 \cdot 0$ & $11 \cdot 1$ \\
\hline Adequate birth weight & 582 & $18 \cdot 6$ & $22 \cdot 8$ & 24.5 & 4.5 & $5 \cdot 0$ & $5 \cdot 0$ & $8 \cdot 1$ & 9.9 & $12 \cdot 0$ & 5.4 & 8.4 & $8 \cdot 8$ \\
\hline Macrosomia & 72 & $23 \cdot 3$ & $32 \cdot 3$ & $18 \cdot 2$ & $10 \cdot 0$ & 0.0 & 0.0 & $10 \cdot 0$ & $16 \cdot 1$ & 0.0 & $3 \cdot 3$ & 9.7 & $18 \cdot 2$ \\
\hline \multicolumn{14}{|l|}{ Delivery mode } \\
\hline Spontaneous delivery & 297 & $21 \cdot 2$ & $28 \cdot 2$ & $27 \cdot 4$ & $6 \cdot 1$ & $6 \cdot 8$ & 4.8 & $10 \cdot 6$ & $12 \cdot 6$ & $17 \cdot 7$ & 4.6 & $9 \cdot 7$ & 6.5 \\
\hline Caesarean delivery & 363 & $16 \cdot 8$ & $20 \cdot 0$ & $26 \cdot 3$ & 4.2 & $2 \cdot 3$ & 4.4 & 5.9 & 8.5 & 11.4 & 5.9 & 8.5 & 11.4 \\
\hline \multicolumn{14}{|l|}{ Mother's education } \\
\hline Middle school or lower & 117 & 24.5 & $18 \cdot 2$ & 35.5 & 7.6 & 3.0 & 6.5 & $13 \cdot 2$ & $12 \cdot 1$ & $25 \cdot 8$ & 3.8 & $6 \cdot 1$ & 6.5 \\
\hline High school and junior college & 326 & $16 \cdot 7$ & $25 \cdot 9$ & $29 \cdot 2^{*}$ & $5 \cdot 3$ & 3.5 & $5 \cdot 2$ & 7.9 & $10 \cdot 3$ & $10 \cdot 3$ & 4.4 & 9.5 & 11.5 \\
\hline College and above & 227 & $19 \cdot 3$ & $24 \cdot 1$ & $15 \cdot 4$ & 3.4 & $5 \cdot 8$ & 1.9 & $5 \cdot 7$ & $10 \cdot 3$ & 9.6 & $6 \cdot 8$ & $9 \cdot 2$ & $7 \cdot 7$ \\
\hline \multicolumn{14}{|l|}{ Monthly income (RMB) } \\
\hline$\leq 2000$ & 175 & $20 \cdot 0$ & $18 \cdot 2$ & 26.5 & 5.0 & 4.6 & $6 \cdot 1$ & $11 \cdot 7$ & $12 \cdot 1$ & $12 \cdot 2$ & 5.0 & 1.5 & $8 \cdot 2$ \\
\hline $2001-4000$ & 238 & $19 \cdot 2$ & 30.9 & $26 \cdot 8$ & 3.0 & 4.4 & $2 \cdot 8$ & $6 \cdot 1$ & $11 \cdot 8$ & $16 \cdot 9^{*}$ & $9 \cdot 1$ & 14.7 & $7 \cdot 0$ \\
\hline$>4000$ & 210 & $18 \cdot 4$ & $22 \cdot 1$ & $27 \cdot 1$ & 6.6 & 3.5 & $4 \cdot 2$ & $9 \cdot 2$ & 7.0 & $10 \cdot 4$ & 1.3 & $9 \cdot 3$ & $16 \cdot 7^{\star}, \dagger$ \\
\hline
\end{tabular}

EBF, exclusive breast-feeding; MBF, mixed feeding with breast milk and infant formula; EFF, exclusive formula-feeding.

Total illness included diarrhoea, respiratory disease, allergic disease and other illness. $n$ varied slightly due to missing data on some variables.

${ }^{*} P$ value from $X^{2}$ test for trend $<0.05$ (EBF, MBF, EFF).

† $P$ value from $\chi^{2}$ test $<0.05$ (EBF, MBF, EFF).

\section{Results}

A total of 1654 infants were included, 671, 501 and 482 aged 0-2.9 months, 3-5.9 months and 6-11.9 months, respectively. The proportions of illness among infants aged 0-2.9 months are shown in Table 1. For total illness, the proportion was $19 \cdot 2 \%$ among EBF infants, $24.1 \%$ among MBF infants and $26 \cdot 3 \%$ among EFF infants. The proportion of diarrhoea ranged from $4 \cdot 2 \%$ to $5 \cdot 1 \%$, which was lower than those of respiratory ( $8.2 \%$ to $13.4 \%)$ and allergic diseases $(5 \cdot 1 \%$ to $9.5 \%)$. The results from the subgroup analyses showed that the proportions of total illness, respiratory disease and allergic disease among EBF infants were lower than those among MBF and EFF infants $(P<0.05)$. Significant difference was not found between MBF and EFF after adjusting for sex, birth weight, delivery mode, mother's education and monthly income.

As shown in Table 2, $41.6 \%$ of EBF infants, $45.6 \%$ of MBF infants and $51.0 \%$ of EFF infants aged 3-5.9 months had been ill. The proportion of diarrhoea ranged from $11.4 \%$ to $18.7 \%$, which was lower than that of respiratory disease $(29.7 \%$ to $37.2 \%)$ but higher than that of allergic disease $(6.9 \%$ to $9.4 \%)$.

Table 3 shows that $67.0 \%$ of EBF infants, $73.4 \%$ of MBF infants and $67.7 \%$ of EFF infants aged 6-11.9 months had been ill. The proportion of diarrhoea ranged from $24.8 \%$ to $30.4 \%$, which was lower than that of respiratory disease ( $48.1 \%$ to $52 \cdot 8 \%$ ) but higher than that of allergic disease (6.5\% to $9.7 \%)$.

The proportions of hospitalization among infants aged 0-11.9 months are shown in Table 4 . A total of $3.9 \%$ to
$8.4 \%$ of infants aged $0-2.9$ months had been hospitalized one or more times. The corresponding proportions were $6.4 \%$ to $8.1 \%$ among those aged 3-5.9 months and $8.4 \%$ to $12 \cdot 1 \%$ among those aged 6-11.9 months. The most frequent reported cause for hospitalization was respiratory disease.

As shown in Table 5, compared with EBF infants, MBF infants had significantly higher odds of having (total) illness and EFF infants had higher odds of having (total) illness and respiratory disease. The odds of having (total) illness, diarrhoea and respiratory disease increased significantly with age. However, such trend was not found in allergic disease. A low birth weight increased the odds of having (total) illness. A high mother's education level decreased the odds of having (total) illness and respiratory disease. A moderate monthly family income (2001-4000 RMB per capita) was related to high odds of having (total) illness and allergic disease.

The result from the multiple logistic regression model showed that feeding pattern was not related to hospitalization (Table 6). Infants aged 6-11.9 months had higher odds (OR $=1.83$; $95 \%$ CI $1 \cdot 13,2 \cdot 98, P=0.014)$ of hospitalization compared with those aged $0-2 \cdot 9$ months. A low birth weight increased the odds of hospitalization $(\mathrm{OR}=9 \cdot 28 ; 95 \%$ CI 3.50, 24.62, $P<0 \cdot 001)$.

\section{Discussion}

Two major findings were obtained in the current study. First, EBF infants had lower odds of having total illness 
Table 2 Feeding mode and proportion of illness in infants aged 3-5.9 months from eight large cities in China, Maternal Infant Nutrition and Growth (MING) study, October 2011-March 2012

\begin{tabular}{|c|c|c|c|c|c|c|c|c|c|c|c|c|c|}
\hline & \multirow[b]{2}{*}{$n$} & \multicolumn{3}{|c|}{ Total illness (\%) } & \multicolumn{3}{|c|}{ Diarrhoea (\%) } & \multicolumn{3}{|c|}{ Respiratory disease (\%) } & \multicolumn{3}{|c|}{ Allergic disease (\%) } \\
\hline & & EBF & MBF & EFF & EBF & MBF & EFF & EBF & MBF & EFF & EBF & MBF & EFF \\
\hline Total & 501 & 41.6 & $45 \cdot 6$ & $51 \cdot 0$ & 11.4 & $18 \cdot 7$ & $13 \cdot 1$ & 29.7 & $26 \cdot 3$ & $37 \cdot 2$ & $7 \cdot 0$ & 9.4 & 6.9 \\
\hline \multicolumn{14}{|l|}{ Sex } \\
\hline Girls & 228 & 43.5 & $45 \cdot 7$ & $50 \cdot 0$ & $12 \cdot 0$ & $22 \cdot 9$ & $9 \cdot 1$ & $29 \cdot 4$ & $27 \cdot 1$ & $36 \cdot 4$ & $8 \cdot 7$ & 2.9 & 4.6 \\
\hline Boys & 272 & $39 \cdot 8$ & $46 \cdot 0$ & 51.9 & $10 \cdot 8$ & $16 \cdot 0$ & $16 \cdot 5$ & $30 \cdot 1$ & $26 \cdot 0$ & 38.0 & 5.4 & 14.0 & 8.9 \\
\hline \multicolumn{14}{|l|}{ Birth weight } \\
\hline Low birth weight & 2 & - & $100 \cdot 0$ & $100 \cdot 0$ & - & $100 \cdot 0$ & $100 \cdot 0$ & - & 0.0 & 0.0 & - & 0.0 & 0.0 \\
\hline Adequate birth weight & 458 & 41.9 & $46 \cdot 4$ & $52 \cdot 2$ & $10 \cdot 2$ & $18 \cdot 3$ & $13 \cdot 0$ & 30.5 & $26 \cdot 8$ & 38.4 & $7 \cdot 8$ & $9 \cdot 2$ & $7 \cdot 3$ \\
\hline Macrosomia & 40 & $41 \cdot 2$ & $35 \cdot 3$ & $16 \cdot 7$ & $23 \cdot 5$ & $17 \cdot 7$ & 0.0 & $23 \cdot 5$ & $23 \cdot 5$ & $16 \cdot 7$ & 0.0 & $5 \cdot 9$ & 0.0 \\
\hline \multicolumn{14}{|l|}{ Delivery mode } \\
\hline Spontaneous delivery & 231 & $41 \cdot 8$ & $41 \cdot 0$ & $50 \cdot 0$ & $6 \cdot 6$ & $19 \cdot 2$ & $12.9 \dagger$ & 33.0 & $23 \cdot 1$ & 33.9 & 4.4 & $10 \cdot 3$ & 6.5 \\
\hline Caesarean delivery & 265 & $42 \cdot 2$ & $50 \cdot 0$ & $51 \cdot 8$ & $15 \cdot 6$ & 18.5 & $13 \cdot 3$ & $26 \cdot 7$ & $29 \cdot 4$ & $39 \cdot 8$ & $10 \cdot 0$ & 8.7 & $7 \cdot 2$ \\
\hline \multicolumn{14}{|l|}{ Mother's education } \\
\hline Middle school or lower & 105 & $55 \cdot 0$ & $54 \cdot 1$ & $57 \cdot 1$ & $25 \cdot 0$ & 18.9 & $14 \cdot 3$ & $42 \cdot 5$ & 43.2 & $39 \cdot 3$ & $12 \cdot 5$ & $2 \cdot 7$ & $0.0^{*}$ \\
\hline High school and junior college & 232 & $43 \cdot 2$ & $45 \cdot 0$ & $45 \cdot 1$ & $7 \cdot 4$ & $22 \cdot 5$ & $11.3 \dagger$ & $33 \cdot 3$ & $21 \cdot 3$ & $33 \cdot 8$ & $3 \cdot 7$ & $7 \cdot 5$ & $7 \cdot 0$ \\
\hline College and above & 164 & $31 \cdot 3$ & $40 \cdot 7$ & $56 \cdot 5^{\star}, \dagger$ & $7 \cdot 8$ & $13 \cdot 0$ & $15 \cdot 2$ & $17 \cdot 2$ & $22 \cdot 2$ & $41 \cdot 3^{*}, \dagger$ & $7 \cdot 8$ & $16 \cdot 7$ & $10 \cdot 9$ \\
\hline \multicolumn{14}{|l|}{ Monthly income (RMB) } \\
\hline$\leq 2000$ & 154 & $43 \cdot 1$ & 41.5 & 39.5 & $17 \cdot 2$ & $17 \cdot 0$ & $9 \cdot 3$ & $31 \cdot 0$ & $30 \cdot 2$ & $32 \cdot 6$ & $8 \cdot 6$ & $9 \cdot 4$ & 0.0 \\
\hline $2001-4000$ & 192 & 43.7 & $47 \cdot 6$ & $56 \cdot 9$ & 8.5 & 17.5 & $17 \cdot 2$ & $33 \cdot 8$ & $27 \cdot 0$ & 37.9 & $7 \cdot 0$ & 7.9 & $10 \cdot 3$ \\
\hline$>4000$ & 136 & 37.0 & $48 \cdot 1$ & $52 \cdot 6$ & 6.5 & $23 \cdot 1$ & $7.9 \dagger$ & 23.9 & $21 \cdot 2$ & $42 \cdot 1$ & $6 \cdot 5$ & 11.5 & $10 \cdot 5$ \\
\hline
\end{tabular}

EBF, exclusive breast-feeding; MBF, mixed feeding with breast milk and infant formula; EFF, exclusive formula-feeding; -, no data exist for this section. Total illness included diarrhoea, respiratory disease, allergic disease and other illness. $n$ varied slightly due to missing data on some variables.

${ }^{*} P$ value from $X^{2}$ test for trend $<0.05$ (EBF, MBF, EFF).

$\dagger P$ value from $\chi^{2}$ test $<0.05$ (EBF, MBF, EFF).

Table 3 Feeding mode and proportion of illness in infants aged 6-11.9 months from eight large cities in China, Maternal Infant Nutrition and Growth (MING) study, October 2011-March 2012

\begin{tabular}{|c|c|c|c|c|c|c|c|c|c|c|c|c|c|}
\hline & \multirow[b]{2}{*}{$n$} & \multicolumn{3}{|c|}{ Total illness (\%) } & \multicolumn{3}{|c|}{ Diarrhoea (\%) } & \multicolumn{3}{|c|}{ Respiratory disease (\%) } & \multicolumn{3}{|c|}{ Allergic disease (\%) } \\
\hline & & EBF & MBF & EFF & EBF & MBF & EFF & EBF & MBF & EFF & EBF & MBF & EFF \\
\hline Total & 482 & $67 \cdot 0$ & $73 \cdot 4$ & $67 \cdot 7$ & $24 \cdot 8$ & $30 \cdot 4$ & $27 \cdot 4$ & $48 \cdot 1$ & $52 \cdot 8$ & $51 \cdot 6$ & $9 \cdot 7$ & 6.5 & 6.5 \\
\hline \multicolumn{14}{|l|}{ Sex } \\
\hline Girls & 208 & $62 \cdot 8$ & 69.7 & 68.0 & $22 \cdot 3$ & 29.2 & $20 \cdot 0$ & $46 \cdot 8$ & 48.3 & 48.0 & $10 \cdot 6$ & $9 \cdot 0$ & $4 \cdot 0$ \\
\hline Boys & 272 & $70 \cdot 3$ & $75 \cdot 8$ & $67 \cdot 6$ & $27 \cdot 0$ & 31.5 & $32 \cdot 4$ & 48.7 & $56 \cdot 5$ & $54 \cdot 1$ & $9 \cdot 0$ & 4.0 & $8 \cdot 1$ \\
\hline \multicolumn{14}{|l|}{ Birth weight } \\
\hline Low birth weight & 5 & $100 \cdot 0$ & $100 \cdot 0$ & $100 \cdot 0$ & 0.0 & $100 \cdot 0$ & 0.0 & $50 \cdot 0$ & 0.0 & 0.0 & 0.0 & 0.0 & 0.0 \\
\hline Adequate birth weight & 436 & $66 \cdot 3$ & 73.5 & 68.6 & $26 \cdot 0$ & $29 \cdot 9$ & $29 \cdot 4$ & $46 \cdot 4$ & 53.9 & $52 \cdot 9$ & $11 \cdot 1$ & $6 \cdot 9$ & $5 \cdot 9$ \\
\hline Macrosomia & 41 & 69.6 & $62 \cdot 5$ & $60 \cdot 0$ & $17 \cdot 4$ & $25 \cdot 0$ & $20 \cdot 0$ & $60 \cdot 9$ & 37.5 & $40 \cdot 0$ & 0.0 & 0.0 & $10 \cdot 0$ \\
\hline \multicolumn{14}{|l|}{ Delivery mode } \\
\hline Spontaneous delivery & 252 & 62.4 & 78.4 & $68.0+$ & 24.8 & 34.3 & $16 \cdot 0$ & $50 \cdot 4$ & $59 \cdot 8$ & 48.0 & 6.4 & $8 \cdot 8$ & 8.0 \\
\hline Caesarean delivery & 226 & 73.4 & 68.5 & $66 \cdot 7$ & $24 \cdot 1$ & $26 \cdot 1$ & 33.3 & 43.0 & $46 \cdot 9$ & $52 \cdot 8$ & 13.9 & 4.5 & $5 \cdot 6^{*}$ \\
\hline \multicolumn{14}{|l|}{ Mother's education } \\
\hline Middle school or lower & 114 & 67.9 & $83 \cdot 3$ & $79 \cdot 0$ & $20 \cdot 8$ & $28 \cdot 6$ & $15 \cdot 8$ & 54.7 & $64 \cdot 3$ & 63.2 & 11.3 & $7 \cdot 1$ & $5 \cdot 3$ \\
\hline High school and junior college & 214 & 71.7 & 71.0 & $79 \cdot 3$ & $25 \cdot 0$ & 28.0 & 44.8 & $52 \cdot 2$ & $52 \cdot 7$ & $58 \cdot 6$ & $15 \cdot 2$ & 6.5 & $6 \cdot 9$ \\
\hline College and above & 154 & 59.0 & $70 \cdot 9$ & $28.6 \dagger$ & $27 \cdot 9$ & 34.2 & $7 \cdot 1$ & $36 \cdot 1$ & $46 \cdot 8$ & $21 \cdot 4$ & 0.0 & $6 \cdot 3$ & $7 \cdot 1$ \\
\hline \multicolumn{14}{|l|}{ Monthly income (RMB) } \\
\hline$\leq 2000$ & 129 & $64 \cdot 2$ & 73.9 & $81 \cdot 3$ & $22 \cdot 4$ & $30 \cdot 4$ & $25 \cdot 0$ & $49 \cdot 3$ & $60 \cdot 9$ & $62 \cdot 5$ & $6 \cdot 0$ & $2 \cdot 2$ & 12.5 \\
\hline $2001-4000$ & 174 & $75 \cdot 4$ & 81.2 & $66 \cdot 7$ & $32 \cdot 3$ & $32 \cdot 9$ & $33 \cdot 3$ & $47 \cdot 7$ & $60 \cdot 0$ & $54 \cdot 2$ & 16.9 & 8.2 & $0 \cdot 0^{*}, \dagger$ \\
\hline$>4000$ & 157 & $60 \cdot 3$ & 63.4 & $55 \cdot 6$ & $20 \cdot 6$ & $28 \cdot 2$ & $22 \cdot 2$ & $45 \cdot 6$ & $42 \cdot 3$ & 33.3 & 5.9 & 8.5 & $5 \cdot 6$ \\
\hline
\end{tabular}

EBF, exclusive breast-feeding; MBF, mixed feeding with breast milk and infant formula; EFF, exclusive formula-feeding.

Total illness included diarrhoea, respiratory disease, allergic disease and other illness. $n$ varied slightly due to missing data on some variables.

${ }^{*} P$ value from $X^{2}$ test for trend $<0.05$ (EBF, MBF, EFF).

$\dagger P$ value from $x^{2}$ test $<0.05$ (EBF, MBF, EFF).

compared with MBF and EFF infants. No statistical association was found between feeding pattern and allergic disease. Second, the proportions of infants having been ill or having had diarrhoea or respiratory disease increased with age in infants, whereas this increase was not observed for allergic disease.
Breast milk provides infants with thousands of components that help build the immune system and protect them from infection ${ }^{(16)}$. Moreover, breast milk, with its optimal amount of nutrients, helps maintain the good nutritional status of infants. The number of EBF infants who had been ill was lower compared with MBF and EFF infants, and this result is 
Table 4 Feeding mode and hospitalization by age in infants aged 0-11.9 months from eight large cities in China, Maternal Infant Nutrition and Growth (MING) study, October 2011-March 2012

\begin{tabular}{|c|c|c|c|c|c|c|c|c|c|c|c|c|c|}
\hline & \multirow[b]{2}{*}{$n$} & \multicolumn{3}{|c|}{ Total illness (\%) } & \multicolumn{3}{|c|}{ Diarrhoea (\%) } & \multicolumn{3}{|c|}{ Respiratory disease (\%) } & \multicolumn{3}{|c|}{ Allergic disease (\%) } \\
\hline & & EBF & MBF & EFF & EBF & MBF & EFF & EBF & MBF & EFF & EBF & MBF & EFF \\
\hline \multicolumn{14}{|l|}{ Hospitalization } \\
\hline $0-2.9$ months & 671 & 3.9 & $6 \cdot 3$ & 8.4 & $0 \cdot 8$ & 0.0 & 0.0 & 1.6 & 2.5 & 3.9 & 0.8 & 0.4 & 0.4 \\
\hline 3-5.9 months & 501 & $8 \cdot 1$ & $6 \cdot 4$ & $6 \cdot 4$ & $1 \cdot 1$ & 0.0 & 0.7 & $3 \cdot 2$ & $4 \cdot 1$ & $2 \cdot 1$ & 0.0 & 0.0 & $0 \cdot 0$ \\
\hline $6-11.9$ months & 482 & $12 \cdot 1$ & 8.4 & $9 \cdot 7$ & 1.0 & 0.0 & 0.0 & $10 \cdot 2$ & 4.2 & $4 \cdot 8^{*}, \dagger$ & 0.0 & 0.0 & $1.6 \dagger$ \\
\hline
\end{tabular}

EBF, exclusive breast-feeding; MBF, mixed feeding with breast milk and infant formula; EFF, exclusive formula-feeding.

Total illness included diarrhoea, respiratory disease, allergic disease and other illness.

${ }^{*} P$ value from $X^{2}$ test for trend $<0.05$ (EBF, MBF, EFF).

† $P$ value from $X^{2}$ test $<0.05$ (EBF, MBF, EFF).

Table 5 Odds ratios for the proportion of illness in infants aged 0-11.9 months from eight large cities in China, Maternal Infant Nutrition and Growth (MING) study, October 2011-March 2012

\begin{tabular}{|c|c|c|c|c|c|c|c|c|}
\hline & \multicolumn{2}{|c|}{ Total illness } & \multicolumn{2}{|c|}{ Diarrhoea } & \multicolumn{2}{|c|}{ Respiratory disease } & \multicolumn{2}{|c|}{ Allergic disease } \\
\hline & AOR & $95 \% \mathrm{Cl}$ & AOR & $95 \% \mathrm{Cl}$ & AOR & $95 \% \mathrm{Cl}$ & AOR & $95 \% \mathrm{Cl}$ \\
\hline \multicolumn{9}{|l|}{ Feeding mode } \\
\hline EBF & 1.00 & Ref. & 1.00 & Ref. & 1.00 & Ref. & 1.00 & Ref. \\
\hline MBF & $1 \cdot 30$ & $1.01,1.68$ & 1.40 & $1.00,1.96$ & $1 \cdot 17$ & $0.89,1.55$ & 1.09 & $0.71,1.68$ \\
\hline EFF & 1.35 & $1.01,1.82$ & 1.02 & $0.66,1.57$ & 1.44 & $1.04,2.00$ & 1.04 & $0.63,1.71$ \\
\hline \multicolumn{9}{|l|}{ Age } \\
\hline $0-2.9$ months & 1.00 & Ref. & 1.00 & Ref. & 1.00 & Ref. & 1.00 & Ref. \\
\hline 3-5.9 months & 2.96 & $2 \cdot 27,3 \cdot 86$ & 3.60 & $2 \cdot 25,5 \cdot 76$ & 3.85 & $2 \cdot 77,5 \cdot 36$ & 1.05 & $0.67,1.63$ \\
\hline $6-11.9$ months & 8.07 & $6 \cdot 07,10 \cdot 71$ & 8.22 & $5 \cdot 26,12 \cdot 85$ & $9 \cdot 19$ & $6 \cdot 61,12 \cdot 76$ & 0.96 & $0.60,1.53$ \\
\hline \multicolumn{9}{|l|}{ Sex } \\
\hline Girls & 1.00 & Ref. & 1.00 & Ref. & 1.00 & Ref. & 1.00 & Ref. \\
\hline Boys & 0.99 & $0.79,1.23$ & 1.06 & $0.78,1.43$ & 1.03 & $0.81,1.31$ & 0.92 & $0.64,1.34$ \\
\hline \multicolumn{9}{|l|}{ Birth weight } \\
\hline Low birth weight & $3 \cdot 24$ & $1.27,8.22$ & $2 \cdot 78$ & $0.83,9.31$ & $2 \cdot 04$ & $0.72,5.75$ & 1.77 & $0.50,6.21$ \\
\hline Adequate birth weight & 1.00 & Ref. & 1.00 & Ref. & 1.00 & Ref. & 1.00 & Ref. \\
\hline Macrosomia & 0.84 & $0.57,1.24$ & 0.73 & $0.41,1.30$ & 0.87 & $0.56,1.34$ & 0.66 & $0.31,1.39$ \\
\hline \multicolumn{9}{|l|}{ Delivery mode } \\
\hline Spontaneous delivery & 1.00 & Ref. & 1.00 & Ref. & 1.00 & Ref. & 1.00 & Ref. \\
\hline Caesarean delivery & 0.98 & $0.78,1.23$ & 1.00 & $0.74,1.35$ & 0.81 & $0.64,1.04$ & 1.30 & $0.89,1.91$ \\
\hline \multicolumn{9}{|l|}{ Mother's education } \\
\hline Middle school or lower & 1.00 & Ref. & 1.00 & Ref. & 1.00 & v & 1.00 & Ref. \\
\hline High school and junior college & 0.75 & $0.56,1.01$ & 0.96 & $0.65,1.43$ & 0.65 & $0.48,0.89$ & $1 \cdot 16$ & $0.68,1.99$ \\
\hline College and above & 0.60 & $0.43,0.84$ & 0.97 & $0.63,1.51$ & 0.47 & $0.33,0.68$ & $1 \cdot 12$ & $0.63,2.00$ \\
\hline \multicolumn{9}{|l|}{ Monthly income (RMB) } \\
\hline$\leq 2000$ & 1.00 & Ref. & 1.00 & Ref. & 1.00 & Ref. & 1.00 & Ref. \\
\hline $2001-4000$ & 1.47 & $1.11,1.94$ & 1.06 & $0.73,1.54$ & $1 \cdot 19$ & $0.88,1.61$ & 1.74 & $1.05,2.87$ \\
\hline$>4000$ & $1 \cdot 10$ & $0.81,1.48$ & 0.85 & $0.56,1.29$ & 0.87 & $0.63,1.22$ & 1.45 & $0.84,2 \cdot 50$ \\
\hline
\end{tabular}

AOR, adjusted odds ratio; EBF, exclusive breast-feeding; MBF, mixed feeding with breast milk and infant formula; EFF, exclusive formula-feeding; Ref., reference category.

Total illness included diarrhoea, respiratory disease, allergic disease and other illness.

consistent with those of previous studies ${ }^{(5-9,17-20)}$. One study reported that full breast-feeding is associated with the lowest illness (diarrhoea, cough, wheeze and vomiting) rates and minimal (less) breast-feeding is not protective ${ }^{(21)}$. Furthermore, a previous multicentre cohort study reported that nonbreast-fed infants have a higher risk of dying compared with predominantly breast-fed (adjusted hazard ratio $=10 \cdot 5$ ) and partially breast-fed infants (adjusted hazard ratio $=2 \cdot 46)^{(18)}$.

Diarrhoeal disease accounted for $11 \%$ of the estimated $7 \cdot 6$ million deaths among under-5s globally in $2010^{(4)}$. In the present study, EBF had a borderline significant protective effect against diarrhoea compared with MBF
$(P=0 \cdot 05)$. Many studies have suggested that breastfeeding, especially EBF, may protect infants against diarrhoeal disease. Dewey et $a l^{(22)}$ reported that the incidence of diarrhoea among breast-fed infants is half that among formula-fed infants in the first year of life. Similar results were found by other studies ${ }^{(8,23-25)}$. The mechanism behind the protective effect of breast milk is that antibodies and living cells in breast milk can prevent infection or attack/kill pathogens that are harmful. Glycans can be recognized by pathogens and become sites of attachment. As a result, the pathogens become harmless and passed out in the faeces. Oligosaccharides and lactose 
Table 6 Odds ratios for total hospitalization in infants aged 0-11.9 months from eight large cities in China, Maternal Infant Nutrition and Growth (MING) study, October 2011-March 2012

\begin{tabular}{|c|c|c|}
\hline & \multicolumn{2}{|c|}{ Total hospitalization } \\
\hline & AOR & $95 \% \mathrm{Cl}$ \\
\hline \multicolumn{3}{|l|}{ Feeding mode } \\
\hline EBF & 1.00 & Ref. \\
\hline MBF & 0.87 & $0.55,1.37$ \\
\hline EFF & 0.86 & $0.50,1.48$ \\
\hline \multicolumn{3}{|l|}{ Age } \\
\hline $0-2.9$ months & 1.00 & \\
\hline 3-5.9 months & $1 \cdot 18$ & $0.70,1.99$ \\
\hline $6-11.9$ months & 1.83 & $1.13,2.98$ \\
\hline \multicolumn{3}{|l|}{ Sex } \\
\hline Girls & 1.00 & Ref. \\
\hline Boys & 1.17 & $0.78,1.76$ \\
\hline \multicolumn{3}{|l|}{ Birth weight } \\
\hline Low birth weight & $9 \cdot 28$ & $3 \cdot 50,24 \cdot 62$ \\
\hline Adequate birth weight & 1.00 & Ref. \\
\hline Macrosomia & 0.63 & $0.27,1.49$ \\
\hline \multicolumn{3}{|l|}{ Delivery mode } \\
\hline Spontaneous delivery & 1.00 & Ref. \\
\hline Caesarean delivery & 0.69 & $0.46,1.04$ \\
\hline \multicolumn{3}{|l|}{ Mother's education } \\
\hline Middle school or lower & 1.00 & Ref. \\
\hline High school and junior college & 1.20 & $0.70,2.06$ \\
\hline College and above & 0.84 & $0.45,1.57$ \\
\hline \multicolumn{3}{|l|}{ Monthly income (RMB) } \\
\hline$\leq 2000$ & 1.00 & Ref. \\
\hline $2001-4000$ & 1.06 & $0.64,1.75$ \\
\hline$>4000$ & $1 \cdot 18$ & $0.69,2.02$ \\
\hline
\end{tabular}

$A O R$, adjusted odds ratio; EBF, exclusive breast-feeding; MBF, mixed feeding with breast milk and infant formula; EFF, exclusive formula-feeding; Ref., reference category.

facilitate the growth of beneficial bacteria, such as bifidobacteria and lactobacilli ${ }^{(16)}$.

Breast-feeding may protect infants against infection with rotavirus $^{(8,9,26)}$. However, it may lead to diarrhoea if the breast milk contains rotavirus ${ }^{(14)}$. A previous study reported that almost half of the acute diarrhoea hospitalization cases among children are caused by rotavirus ${ }^{(27)}$. Thus, healthy breast milk is the key factor in protecting infants' health.

Respiratory disease is another leading disease among infants. In the current study, respiratory disease was the most common reported illness. EBF also exhibited a protective effect against this disease. Our results are consistent with those of other findings ${ }^{(5,7,17,25)}$. Soto-Ramirez et $a l^{(28)}$ found that compared with direct breast milk, any feeding pattern that includes formula or bottled breast milk seems to be a moderate risk for cough or wheezing episodes in the first 12 months of life. The protective effect of breast milk is biologically plausible. On one hand, breast milk has a direct action against pathogenic agents and an indirect action that is mediated by its capacity to affect the infant's physiology by modulating mucosal immune responses ${ }^{(29)}$. On the other hand, formulas lack lipids and immune-active components that may help fight foreign pathogens (e.g. viruses, bacteria) and in turn help protect against the development of respiratory diseases, such as viral upper respiratory infections.
The development of allergic disease depends on numerous variables and reflects the interaction between genetic factors and environmental exposure. However, whether or not breast milk prevents or reduces the incidence of allergic diseases remains unclear. Particular elements in breast milk are thought to function as protective agents in infants to prevent the development of allergies, whereas others may function in a different way $^{(30)}$. In the present study, a significant relationship was not observed between allergic diseases and feeding pattern and the proportion of patients who developed allergic diseases did not increase with age. Previous studies suggested that prolonged breast-feeding and $\mathrm{EBF}$ reduce the risk of eczema and allergic diseases in children $^{(10,12,31-33)}$. However, other studies failed to confirm this finding or even found that breast-feeding is associated with an increased risk for childhood asthma in the presence of maternal asthma ${ }^{(11,13,34-36)}$. For example, Kramer and Kakuma ${ }^{(37)}$, in their meta-analysis on optimal duration of $\mathrm{EBF}$, reported that $\mathrm{EBF}$ for 6 months has no long-term impact on allergic disease development $v$. those fed for 3-4 months with continued mixed breast-feeding thereafter. In addition, a study showed that the levels of soluble CD14, total IgA and transforming growth factor- $\beta 1$ (TGF- $\beta 1$ ) in breast milk at either day 7 or day 28 are not associated with subsequent development of eczema or atopic sensitization during the first year of life ${ }^{(38)}$. Similarly, but not totally, another study reported that maternal serum and whey levels of IL- 5 and IL-13 are risk markers for asthma-like symptoms, whereas whey IgA and TGF- $\beta 1$ seem to have a protective effect during infancy ${ }^{(13)}$. The composition of human milk can be affected by the diet of lactating women ${ }^{(39-41)}$. Thus, speculating that the exclusion of peanuts, egg, fish and other potentially 'antigenic' foods in the maternal diet while breast-feeding may reduce the risk of allergy in infants is reasonable. Several studies found a protective effect on the development of atopic dermatitis in infants when mothers avoided these foods ${ }^{(42,43)}$. However, recent studies have concluded that evidence is inadequate to advise women to avoid specific food items during pregnancy or breast-feeding to protect their children from allergic diseases, such as eczema and asthma ${ }^{(44)}$.

These controversial results on the protective effect of breast-feeding on infant allergy from different studies may be attributed to diverse methods, subjects and definitions of $\mathrm{EBF}$, as well as to the fact that allergic disease is related to heredity, family history and other inducing factors, as previously reported. Previous studies reported that breastfeeding increases the risk for atopy among boys with paternal atopy (OR=7.39; 95\% CI, 2.21, 24.66) compared with non-breast-fed boys with paternal atopy, and the result was similar among girls $(\mathrm{OR}=3 \cdot 13 ; 95 \% \mathrm{CI}, 1 \cdot 20,8 \cdot 14)^{(34)}$. Conversely, other studies found that the children who benefited the most from breast-feeding are those with a parental history of atopic disease independent of maternal asthma ${ }^{(10,31)}$. Thus, whether breast milk has a protective 
function or is a risk factor may be related to the heredity of infants. However, further studies are recommended.

In the present study, mother's education was associated with the proportions of total illness and respiratory disease during infancy. A high level of mother's education indicated a low proportion of total illness and respiratory disease. This result is consistent with those of another Chinese study ${ }^{(45)}$. Usually, mothers are the main caregivers in China. Educated mothers have more knowledge of baby care and they have more access to acquire new and scientific parenting skills than those with less education. In addition, mothers with a high level of education usually have greater financial capacity, thereby allowing them to fully reap the benefits of education, provide their children with more hygienic living environments and obtain greater access to health care ${ }^{(11)}$. Furthermore, previous studies reported that the level of mother's education is related with non-healthy behaviours, such as no hair washing, no showering, no teeth cleaning and no indoor air ventilation during the first days after childbirth or even the entire period of puerperium, and maternity blues. Those with a low level of education have a higher incidence of practising non-healthy behaviours and maternity blues ${ }^{(46,47)}$, which may result in an unfavourable effect on infants' health care.

In the current study, among all the diseases except allergic disease, EBF had a protective effect compared with $\mathrm{MBF}$ and EFF. Breast milk is superior to formulas not only in nutrients, immune components and psychological benefits, but also in safety, convenience and feasibility. Infants can make full use of breast milk with their special digestive system in EBF. Thus, EBF should be encouraged for at least 4 to 6 months in infants. However, the extent of the favourable effect of MBF on infants' health remains unknown. More studies are needed to investigate this issue.

The limitations of our study should be noted. First, for infants aged 6 months and below, they were classified as $\mathrm{EBF}, \mathrm{MBF}$ or EFF based on how they were fed since they were born because we were unable to obtain information until they were 6 months old. Second, we focused on the proportion of the infants who had a specific disease and had been hospitalized for treatment since they were born but could not obtain information on the prevalence. Third, recall bias may exist. Caregivers may forget to report slight illnesses, such as cough and sneeze, or they may be not able to distinguish between respiratory infectious morbidity and allergic disease, if not diagnosed by a doctor. However, most parents/guardians are sensitive to infants' health, thereby helping in minimizing recall bias. Fourth, no causal relationships are precisely delineated from a cross-sectional study.

\section{Conclusion}

In conclusion, EBF had a protective effect against total illness in Chinese infants. Although we did not find the same result for allergic diseases, breast-feeding, especially $\mathrm{EBF}$, is recommended for babies. Healthy breast milk is the best food to be given to infants worldwide because of its nutritive contents, protective qualities against common diseases, immunological protection against long-term chronic diseases and the psychological benefits.

\section{Acknowledgements}

Financial support: This research project was part of the larger MING study, sponsored by Nestlé Nutrition Institute of China and Nestlé Research Center. This research project was a collaborative work between Peking University and Nestlé. The views and opinions expressed in this manuscript are those of the authors and do not necessarily reflect the opinions and recommendations of Nestlé. Conflict of interest: None declared. Authorship: X.Y., W.L. and P.W. contributed to the conception and design of the study. Y.Z. contributed to the conception and design of the study and critical revisions of the manuscript. L.C. contributed to the analysis and interpretation of data and drafted the manuscript. P.Y. contributed to interpretation of data and drafted the manuscript. Ethics of human subject participation: This study was conducted according to the guidelines laid down in the Declaration of Helsinki and all procedures involving human subjects were approved by the Medical Ethics Research Board of Peking University (approval number IRB00001052-11042). Written informed consent was acquired from all infants' legal guardian.

\section{References}

1. Section on Breastfeeding (2005) Breastfeeding and the use of human milk. Pediatrics 129, e827-e841.

2. Xi W (2002) The composition and functions of human milk. Foreign Med Sci 13, 243-245.

3. Oddy WH (2001) Breastfeeding protects against illness and infection in infants and children: a review of the evidence. Breastfeed Rev 9, 11-18.

4. Lopez-Alarcon M, Villalpando S \& Fajardo A (1997) Breastfeeding lowers the frequency and duration of acute respiratory infection and diarrhea in infants under six months of age. J Nutr 127, 436-443.

5. Cushing AH, Samet JM, Lambert WE et al. (1998) Breastfeeding reduces risk of respiratory illness in infants. $\mathrm{Am} \mathrm{J}$ Epidemiol 147, 863-870.

6. Lin F, Chen F, Wei H et al. (2001) Follow-up study of 100 cases of breast-feeding and bottle-feeding infants. J Clin Pediatr 19, 177-189.

7. Oddy WH, Sly PD, de Klerk NH et al. (2003) Breast feeding and respiratory morbidity in infancy: a birth cohort study. Arch Dis Child 88, 224-228.

8. Gu Z \& Shen H (2010) The effects of breast feeding or bottle feeding on infants with rotavirus diarrhea. J Clin Pediatr 28, $57-60$.

9. Dey SK, Chisti MJ, Das SK et al. (2013) Characteristics of diarrheal illnesses in non-breast fed infants attending a large urban diarrheal disease hospital in Bangladesh. PLoS One 8, e58228. 
10. van Odijk J, Kull I, Borres MP et al. (2003) Breastfeeding and allergic disease: a multidisciplinary review of the literature (1966-2001) on the mode of early feeding in infancy and its impact on later atopic manifestations. Allergy 58, 833-843.

11. Kramer MS (2011) Breastfeeding and allergy: the evidence. Ann Nutr Metab 59, Suppl. 1, 20-26.

12. Ehlayel MS \& Bener A (2008) Duration of breast-feeding and the risk of childhood allergic diseases in a developing country. Allergy Asthma Proc 29, 386-391.

13. Soto-Ramirez N, Karmaus W, Yousefi M et al. (2012) Maternal immune markers in serum during gestation and in breast milk and the risk of asthma-like symptoms at ages 6 and 12 months: a longitudinal study. Allergy Asthma Clin Immunol 8, 11.

14. Ye J (2011) The effects of different feeding patterns on infants with rotavirus diarrhea. Chin Prim Health Care 25 40-41.

15. Jihong C, Xiaolan Z \& Shiyuan W (2008) Effects of different feeding patterns on infant eczema. Matern Child Health Care China 23, 1660-1661.

16. Gribble KD (2011) Mechanisms behind breastmilk's protection against, and artificial baby milk's facilitation of, diarrhoeal illness. Breastfeed Rev 19, 19-26.

17. Pardo-Crespo R, Perez-Iglesias R, Llorca J et al. (2004) Breast-feeding and risk of hospitalization for all causes and fever of unknown origin. Eur J Public Health 14, 230-234.

18. Bahl R, Frost C, Kirkwood BR et al. (2005) Infant feeding patterns and risks of death and hospitalization in the first half of infancy: multicentre cohort study. Bull World Health Organ 83, 418-426.

19. Liu X (2013) The relationship of feeding pattern and infancy common disease. Chin Remedies Clin 13, 67-68.

20. Mihrshahi S, Oddy WH, Peat JK et al. (2008) Association between infant feeding patterns and diarrhoeal and respiratory illness: a cohort study in Chittagong, Bangladesh. Int Breastfeed J 3, 28.

21. Raisler J, Alexander C \& O'Campo P (1999) Breast-feeding and infant illness: a dose-response relationship? Am J Public Health 89, 25-30.

22. Dewey KG, Heinig MJ \& Nommsen-Rivers LA (1995) Differences in morbidity between breast-fed and formulafed infants. J Pediatr 126, 696-702.

23. Brown KH, Black RE, Lopez DRG et al. (1989) Infant-feeding practices and their relationship with diarrheal and other diseases in Huascar (Lima), Peru. Pediatrics $\mathbf{8 3}$ 31-40.

24. Shah SM, Yousafzai M, Lakhani NB et al. (2003) Prevalence and correlates of diarrhea. Indian J Pediatr 70, 207-211.

25. Arifeen S, Black RE, Antelman G et al. (2001) Exclusive breastfeeding reduces acute respiratory infection and diarrhea deaths among infants in Dhaka slums. Pediatrics 108, E67.

26. Shen H, Li H, Zhang J et al. (2003) Effects of breast feeding on infants with rotavirus diarrhea. Sh J Prev Med 15, 175-176.

27. Nakawesi JS, Wobudeya E, Ndeezi G et al. (2010) Prevalence and factors associated with rotavirus infection among children admitted with acute diarrhea in Uganda. BMC Pediatr 10, 69.

28. Soto-Ramirez N, Karmaus W, Zhang H et al. (2013) Modes of infant feeding and the occurrence of coughing/wheezing in the first year of life. J Hum Lact 29, 71-80.

29. Lanari M, Prinelli F, Adorni F et al. (2013) Maternal milk protects infants against bronchiolitis during the first year of life. Results from an Italian cohort of newborns. Early Hum Dev 89, Suppl. 1, S51-S57.

30. Friedman NJ \& Zeiger RS (2005) The role of breast-feeding in the development of allergies and asthma. J Allergy Clin Immunol 115, 1238-1248.

31. Kull I, Wickman M, Lilja G et al. (2002) Breast feeding and allergic diseases in infants - a prospective birth cohort study. Arch Dis Child 87, 478-481.

32. Bener A, Ehlayel MS, Alsowaidi S et al. (2007) Role of breast feeding in primary prevention of asthma and allergic diseases in a traditional society. Eur Ann Allergy Clin Immunol 39, 337-343.

33. Huang H, Zhang F, Hang J et al. (2013) Cohort study of 684 pairs of mother-and-child allergic diseases. Chin J Pediatr 51, 168-171.

34. Mandhane PJ, Greene JM \& Sears MR (2007) Interactions between breast-feeding, specific parental atopy, and sex on development of asthma and atopy. J Allergy Clin Immunol 119, 1359-1366.

35. McNiel ME, Labbok MH \& Abrahams SW (2010) What are the risks associated with formula feeding? A re-analysis and review. Birth 37, 50-58.

36. Zhang Y, Wang L \& Chen G (2013) Meta-analysis of correlation between breastfeeding and childhood asthma. J Clin Pediatr 31, 186-189.

37. Kramer MS \& Kakuma R (2012) Optimal duration of exclusive breastfeeding. Cochrane Database Syst Rev 8, CD003517.

38. Ismail IH, Licciardi PV, Oppedisano F et al. (2013) Relationship between breast milk sCD14, TGF- $\beta 1$ and total IgA in the first month and development of eczema during infancy. Pediatr Allergy Immunol 24, 352-360.

39. Emmett PM \& Rogers IS (1997) Properties of human milk and their relationship with maternal nutrition. Early Hum Dev 49, Suppl, S7-S28.

40. Alam DS, van Raaij JM, Hautvast JG et al. (2010) Effect of dietary fat supplementation during late pregnancy and first six months of lactation on maternal and infant vitamin A status in rural Bangladesh. J Health Popul Nutr 28, 333-342.

41. Lonnerdal B (1986) Effects of maternal dietary intake on human milk composition. J Nutr 116, 499-513.

42. Lovegrove JA, Hampton SM \& Morgan JB (1994) The immunological and long-term atopic outcome of infants born to women following a milk-free diet during late pregnancy and lactation: a pilot study. BrJ Nutr $\mathbf{7 1}$, 223-238.

43. Ip S, Chung M, Raman G et al. (2007) Breastfeeding and maternal and infant health outcomes in developed countries. Evid Rep Technol Assess (Full Rep) issue 153 $1-186$.

44. Kramer MS \& Kakuma R (2014) Cochrane in context: Maternal dietary antigen avoidance during pregnancy or lactation, or both, for preventing or treating atopic disease in the child. Evid Based Child Health 9, 484-485.

45. Jie L, Tao Y, Yimin L et al. (2008) Epidemiological investigation on risk factors of infant eczema. Matern Child Health Care China 21, 3025-3026.

46. Wu J, Mei S, Xu Q et al. (2012) A study of 545 cases of the related factors of postpartum depression. Matern Child Health Care China 27, 889-891.

47. Xue M, Zhang S, Li Y et al. (2011) Health care knowledge attitude and practice of puerperal women and the influencing factors. Matern Child Health Care China 26, 794-797. 